\title{
Comparison of total antioxidant capacity (TAC)'s patients of chronic kidney disease undergoing hemodialysis and non-hemodialysis
}

\author{
Zaky Firmawan El-Hakim ${ }^{1}{ }^{\oplus}$, Mochammad Thaha ${ }^{2}{ }^{\oplus}$, Yetti Hernaningsih ${ }^{3}$ \\ ${ }^{1}$ Medical Program, Faculty of Medicine, Universitas Airlangga, Surabaya, Indonesia, ${ }^{2}$ Department of Internal \\ Medicine, Universitas Airlangga, Universitas Airlangga Academic Hospital, Surabaya, Indonesia, \\ ${ }^{3}$ Department of Clinical Pathology, Faculty of Medicine, Universitas Airlangga, Surabaya, Indonesia
}

\begin{tabular}{l}
\hline Article Info \\
\hline Article history: \\
Received Nov 10, 2019 \\
Revised Apr 30, 2020 \\
Accepted May 18, 2020 \\
Published Jul 1, 2020 \\
\hline
\end{tabular}

\section{Keywords:}

Chronic kidney disease

Total antioxidant capacity

Hemodialysis

Antioxidant

Oxidative stress

\begin{abstract}
Background: Total antioxidant capacity (TAC) is one of the biomarkers to determine a person's health condition through antioxidant and oxidant activity or oxidative stress so that it can help in determining the therapy needed. However, currently, TAC levels in patients with chronic kidney disease (CKD) undergoing hemodialysis (HD) and non-hemodialysis (non-HD) are still controversial, so further research is needed. Objective: To analyze the comparison of TAC in HD and non-HD CKD patient. Materials and Methods: This was an analytical cross-sectional study with clinical observation in CKD patient. A total of $71 \mathrm{CKD}$ patients consisting of $28 \mathrm{CKD}$ HD patients and 43 non-HD CKD patients were enrolled in this study. Data about the characteristics of the subjects were taken using the anamnesis method, while data about TAC were taken using the colorimetric method with COBAS C-501 0837-19 series and 0835-13 series to measure TAC levels in the serum of CKD patients. The data that has been obtained were analyzed using independent sample t-tests. Results: TAC levels were significantly higher in CKD patients undergoing HD compared to non-HD CKD patients ( $\mathrm{p}<0.05)$. There are data on albumin as an antioxidant and MDA as an oxidative stress biomarker that affects TAC levels. Conclusion: TAC levels found to be higher in hemodialysis CKD patient compared to non-hemodialysis CKD patient.
\end{abstract}

(c) (i)

\section{Corresponding Author:}

Mochammad Thaha

Department of Internal Medicine, Faculty of Medicine, Universitas Airlangga

Universitas Airlangga Academic Hospital,

Jl. Dharmahusada Permai, Mulyorejo, Kec. Mulyorejo, Surabaya 60115, East Java, Indonesia mochthaha@fk.unair.ac.id

\section{BACKGROUND}

Total antioxidant capacity (TAC) is a serum examination that is used to measure the capacity and total antioxidant activity found in the body, this examination will later provide data of levels of total antioxidant capacity which is one of the important biomarkers in monitoring the person's health condition. In patients with chronic kidney disease (CKD), structural damage and kidney dysfunction occur, this can trigger the emergence of oxidative stress caused by increased oxidant activity and decreased antioxidant systems which will later have an impact on the TAC (Putri \& Thaha, 2014). A 
number of studies have reported TAC levels of CKD patients who undergo hemodialysis (HD) and nonhemodialysis (non-HD) that are quite varied and controversial (Mayer et al., 2003; Montazerifar et al., 2012). In addition, in Indonesia, especially in Surabaya, there is still no data on TAC CKD patients who undergo HD and non-HD.

\section{OBJECTIVE}

The purpose of this study was to analyze the differences in TAC CKD patients who undergo HD and non-HD as well as to obtain data on TAC levels in CKD HD and non-HD patients.

\section{MATERIALS AND METHODS}

This study was a cross sectional analysis study with clinical observations in CKD patients. A total of 71 CKD patients consisting of 28 CKD patients who undergo HD procedures and 43 non-HD CKD patients in Universitas Airlangga Hospital, Royal Surabaya Hospital, and Premier Surabaya Hospital. Subjects were selected using inclusion criteria namely, adult subjects, aged $\geq 21$ years; diagnosis of CKD is based on the medical record of the subject; no clinical signs of infection (afrebis in the last 3 days); no signs of dehydration; no clinical signs of excess fluid causing edema; hemodialysis subjects were categorized as CKD patients who had undergone hemodialysis for $\geq 3$ months and were stable; while for the exclusion criteria, namely, the patient received Fe preparation. Then, the data was collected using anamnesis method for patient profiles in the form of age, history of disease, history of dialysis, and history of subject habits. To measure TAC levels in the serum of CKD patients, the colorimetric method with the COBAS C-501 0837-19 series and 0835-13 series was used to obtain data on the levels of total antioxidant capacity of the subjects. In this study, it also measured levels of albumin which is an antioxidant and malondialdehyde (MDA) which is a patient's oxidative stress biomarker as a comparison between antioxidant and oxidant levels to strengthen data on total antioxidant capacity. Furthermore, the data collected from several examinations would be analyzed for comparison using the Statistical Package for the Social Sciences (SPSS) application. To analyze the TAC comparison of CKD HD and non-HD patients, we conducted independent sample t- test.

\section{RESULTS}

\section{General characteristics and profile of research subjects}

Table 1 presented data on general characteristics and profile of research subjects which contained gender, age, CKD stage, history of disease, as well as history of research subjects' habits.

Table 1. Characteristics of research subjects

\begin{tabular}{|c|c|c|c|}
\hline \multicolumn{2}{|c|}{ Variables } & \multirow{2}{*}{$\frac{\mathrm{HD}}{16(22.5)}$} & \multirow{2}{*}{$\frac{\text { Non-HD }}{25(35.2)}$} \\
\hline Gender [n (\%)] & Male & & \\
\hline & Female & $12(17)$ & $18(25.3)$ \\
\hline \multirow[t]{5}{*}{ Age $[\mathrm{n}(\%)]$} & Early adulthood (26-35years) & 2 & - \\
\hline & Late adulthood (36-45years) & 2 & - \\
\hline & Early elderly (46-55 years) & 8 & 13 \\
\hline & Late elderly (56-65 years) & 11 & 25 \\
\hline & Seniors ( $\geq 65$ years) & 10 & 4 \\
\hline \multirow[t]{5}{*}{ CKD Stage [n (\%)] } & 5 & $28(39.5)$ & $2(2.8)$ \\
\hline & 4 & - & $16(22.5)$ \\
\hline & 3 & - & $17(24)$ \\
\hline & 2 & - & $5(7)$ \\
\hline & 1 & - & $3(4.2)$ \\
\hline \multirow[t]{5}{*}{ History of Disease [n (\%)] } & Hypertension & $27(38)$ & $38(53.5)$ \\
\hline & Diabetes Mellitus & $16(22.5)$ & $40(56.3)$ \\
\hline & Dyslipidemia & $16(22.5)$ & $27(38)$ \\
\hline & Heart Failure & $15(21.1)$ & $9(12.7)$ \\
\hline & Coronary Heart Disease & $2(2.8)$ & $3(4.2)$ \\
\hline \multirow[t]{2}{*}{ History of Habits [n (\%)] } & Smoking & $7(9.9)$ & $10(14.1)$ \\
\hline & Alcohol Consumption & $2(2.8)$ & $1(1.4)$ \\
\hline
\end{tabular}


Table 2. Levels of total antioxidant status

\begin{tabular}{llcccc}
\hline & Category & $\mathrm{N}$ & Average & $\begin{array}{c}\text { Lowest } \\
\text { limit }\end{array}$ & $\begin{array}{c}\text { Highest } \\
\text { limit }\end{array}$ \\
\hline TAC & HD & 28 & 2.2032 & 1.61 & 2.5 \\
& Non-HD & 43 & 1.6814 & 1.52 & 1.71 \\
\hline
\end{tabular}

Table 2 presents data in the form of TAC levels in patients with chronic kidney disease who undergo $\mathrm{HD}$ and non-HD procedures. The results of independent sample test conducted on these data indicate that TAC levels were significantly higher in CKD patients undergoing HD compared to CKD non-HD patients ( $\mathrm{p}<0.05)$. From laboratory tests conducted on patient's TAC levels, there are also data on albumin and MDA which shows one of the antioxidant components and oxidative stress biomarkers found in the subject's serum (Thaha, 2017).

\section{DISCUSSION}

\section{Total Antioxidant status levels in chronic kidney disease patients undergoing hemodialysis and non-hemodialysis}

As shown in Table 1, some subjects in this study have a history of diseases such as hypertension, diabetes mellitus, dyslipidemia, heart failure, and coronary heart disease. This is in accordance with research by Putri \& Thaha (2014) and Sundaram et al. (2014) that CKD is influenced by risk factors in the form of the disease. Some subjects also have bad habits such as smoking and consuming alcoholic drinks. According to Akinyemi \& Ajayi (2017), this can be a trigger or even worsen the condition of CKD patients.

The results of the analysis in this study showed that CKD patients who underwent HD procedures had a significantly higher TAC than CKD patients who did not undergo HD. procedures. This is caused by antioxidant activity and oxidative stress (Buico, et al., 2009); in addition, indirectly TAC is also influenced by a person's history of disease related to oxidative stress itself (Putri \& Thaha, 2014; Sundaram, et al., 2014). In the case of this study, the subjects were divided into 2 groups, according to Sundaram, et al. (2014) TAC levels of individuals who do not undergo HD will be lower than healthy individuals due to factors of oxidative stress arising from a history of CKD in the group's subjects. On the other hand, TAC in the group of CKD patients who underwent the HD procedure was influenced by a history of CKD, and also affected by the HD procedure done, it was strengthened by Liakopoulos, et al., (2017) who stated that stress oxidative that appear even in the early stages of CKD will increase frequently with worsening the kidney's function, it will also be worsen by HD procedures done.

\section{Factors affecting total antioxidant status levels in chronic kidney disease patients}

Several studies have suggested that oxidative stress in individuals with CKD is influenced by several factors such as increased oxidation activity, decreased protective mechanisms of oxidants, diabetes mellitus type 2, dyslipidemia, increased phagocytic activity, inflammation, and failure of ROS removal, and factors associated with uremia (Putri \& Thaha, 2014; Sundaram, et al. 2014; LadiAkinyemi \& Ajayi, 2017).

An article written by Tangvarasittichai (2015) stated that oxidative stress increases in metabolic syndrome and type 2 diabetes and is most likely a cause of developing cardiovascular disease, diabetes mellitus type 2, and other diabetes complications. Increased oxidative stress is a damaging factor that leads to insulin resistance, dyslipidemia, $\beta$-cell dysfunction, impaired glucose tolerance; oxidative stress, hyperglycemia. This causes decreased insulin production, fasting hyperglycemia and ultimately causes the development of type $2 \mathrm{DM}$ which is getting worse.

Sundaram et al. (2014) stated that hyperglycemia can cause vascular damage through several mechanisms, and there are four mechanisms; all of these mechanisms will lead to the formation of oxidative stress that comes from inflammation, and coagulopathy which can also cause atherosclerosis. In the body of patients with CKD, there will be an accumulation of Advanced Glycation End-products (AGEs) which are biomarkers of oxidative stress, a product of carbohydrate oxidation.

Dyslipidemia has a relationship with the incidence of CKD and the prognosis of CKD, through several inflammatory mechanisms and increased oxidative stress, which will lead to endothelial damage and atherosclerosis (Sheen \& Sheu, 2011). According to Delaney (2006), an increase in serum triglycerides 
(TGL), which is the predominance of very low-density lipoprotein (VLDL) and intermediate-density lipoprotein (IDL), will lead to hypertriglyceridemia and low high density lipoprotein (HDL).

Research conducted by Tangvarasittichai, S. (2015) showed that in the group of subjects with higher lipid concentrations, there was an increase in the concentration of malondialdehyde (MDA). Malondialdehyde is a biomarker of oxidative stress which is a by-product of lipid peroxidation. In the early stages of dyslipidemia, the cytotoxic effects of ROS are likely to have affected the body, then MDA will continue to increase along with the progression of the disease, and there will be an overproduction of free radicals causing lipid peroxidation and cell damage due to oxidation (Kattoor et al., 2017). All of these changes will eventually lead to inflammation, oxidative stress, and coagulopathy, and can cause atherosclerosis which will further aggravate the patient's condition (Goldberg, 2000). In this study, it was proven by significantly higher MDA levels in CKD patients who did not undergo HD compared to patients who underwent HD (Thaha, 2017).

Inflammation and oxidative stress are two things that are interrelated with each other and are factors in the occurrence of kidney failure triggered by mechanisms such as endothelial dysfunction and general complications such as cardiovascular disease and death; as previously known that CKD is an inflammatory disease or inflammation where ROS formation is the center of several inflammatory diseases (Sundaram, et al., 2014; Liakopoulos, et al., 2017).

Several studies have shown that oxidative stress can cause inflammation, CKD patients are in the stage of chronic inflammation which will lead to increased oxidative stress through the activation mechanisms of inflammatory cells such as polymorphonuclear (PMNs) and monocytes; these cells will increase the secretion of NADPH oxidase and myeloperoxidase (MPO) which will cause an increase in ROS (Cohen \& Hörl, 2012; Putri \& Thaha, 2014; Sundaram, et al., 2014; Liakopoulos, et al., 2017).

In patients suffering from CKD in a state of uremia, LDL will be more easily oxidized compared to normal people. Low density oxidized lipoprotein (Ox-LDL) is a molecule that can trigger inflammatory processes in blood vessels, this will lead to the expression of leukocyte adhesion molecules and binding to inflammatory cells in the circulation, this shows that uremia indirectly affects the formation of stress oxidative via inflammatory pathways (Putri \& Thaha, 2014). Some proofs presented by Sundaram MS (2014) also showed that antioxidant enzymes will change in response to decreased kidney function and will get worse in patients with uremia.

In addition, many studies state that aging, antioxidant diets, smoking, and alcohol consumption can also influence oxidative stress which will also affect TAS levels (Liguori, et al., 2018; Thaha et al. 2010; van der Vaart, et al., 2005; Nsonwu-Anyanwu, et al., 2018; Almansa et al., 2013; Flores-Bellver et al., 2014).

\section{Factors affecting total antioxidant status of chronic kidney disease patients who underwent hemodialysis}

In research subjects undergoing HD procedures, oxidative stress is also influenced by HD procedure. Basically HD will indeed provide benefits for individuals suffering from CKD, especially those who have reached terminal stage (Berns, 2017). Nevertheless, behind these benefits, HD also has side effects caused by compatibility of dialyzer membranes, dietary restrictions of vegetables and fruits during HD procedures, loss of antioxidants, and accumulation of oxidative products during HD procedures (Sundaram, et al., 2014; Liakopoulos, et al., 2017).

Bioincompatibility in HD systems has an important role in the production of ROS through the activation of neutrophils PMN, there are two components that greatly influence the formation of oxidative stress in HD procedures, namely dialyzer membrane and the amount of endotoxins in the dialysate. At the time of dialysis process, there is a possibility of disappearance of vitamins and antioxidants, this is proven by the findings that showed plasma vitamin $\mathrm{E}$ and selenium are reduced in patients undergoing HD procedures; but also found significant deficiencies and downregulation of glutathione peroxidase, copper-zinc superoxide dismutase, and manganese superoxide dismutase in kidney disorders (Kuo \& Tarng, 2010; Sundaram, et al. 2014). Hemodialysis (HD) which uses cellulose and semi-cellulose membranes also contributes to increasing oxidants through the mechanism of activating the complement pathway and by stimulating inflammation which then leads to increased oxidant production and ultimately will increase oxidative stress (Coombes \& Fassett, 2012; Putri \& Thaha, 2014).

In addition to bioincompatibility in dialyzer membrane, it is known that white blood cells and platelet activation that lead to oxidative responses are significantly increased by dialysate microbial 
contaminants. Even if a small amount of dialysate is contaminated, it will worsen the biocompatibility of HD therapy and will also aggravate amyloidosis, inflammation, atherosclerosis caused by HD, and accumulation of oxidative products will form (Labriola \& Jadoul, 2017).

However, oxidative stress that is formed during HD process can be reduced by decreasing the activity of inflammatory cells and eliminating inflammatory mediators. To reduce the activity of inflammatory cells can be done by using synthetic biocompatible membranes such as polysulfone and using ultrapure dialysate, there is increasing evidence that the use of ultrapure dialysate in HD patients can reduce the level of serum inflammatory mediators, $\beta 2$-microglobulin, carbonyl, and oxidative stress biomarkers and increase anemia status (Liakopoulos, 2017). Meanwhile, to eliminate inflammatory mediators, hemolipodialysis can be done, namely by using a dialysis membrane that has been coated with vitamin E and the use of reduced water electrolysis (Tajbakhsh, et al., 2017). According to Putri \&Thaha (2014) vitamin E coatings performed on cellulose membranes can reduce oxidative stress and prevent damage to endothelial function caused by HD procedures.

\section{Comparison of total antioxidant status of chronic kidney disease patients who underwent hemodialysis and non- hemodialysis}

In this study higher TAS levels in HD patients were caused by accumulation of endogenous antioxidants that were formed. Urine albumin levels of HD patients are higher than those of non-HD patients and normal people, where the normal limit of urine albumin in the body is <30 $\mathrm{mg}$ ( 24 hour urine), while serum albumin levels in both groups of subjects are still within normal limits, there are even some subjects who have serum albumin levels below normal, where the normal limit of serum albumin is $3.5-5.5 \mathrm{~g} / \mathrm{dl}$ (Medscape, 2018; NIDDK, 2018).

High levels of albumin in the urine are caused by inflammation that occurs in the glomerular kidney, this condition is often called glomerulonephritis or nephritis; moreover, diabetes and high blood pressure are also the main risk factors for the occurrence of albuminuria because both of these can also interfere with kidney function; older age, weight gain and certain races can also increase the risk of albuminuria; Cardiovascular disease is also believed to have a relationship with albuminuria (Kidney Health Australia, 2015). Thus, the data obtained about history of disease suffered by research subjects such as diabetes mellitus, cardiovascular disease is stronger in proving its effect on the levels of antioxidant status indirectly in CKD patients.

In addition to high albumin levels, high TAS levels most likely were also affected by gout, nevertheless in this study no gout was examined. High levels of uric acid in the body are known as hyperuricemia, this often happens in individuals with CKD. Hyperuricemia is caused by decreased ability to remove uric acid by the kidneys; data obtained by Filiopoulos et al. (2012) showed that hyperuricemia and hypertension had a strong association with cardiovascular disease, CKD progression, and mortality where oxidative stress had an impact. The main benefit provided by gout is its ability to act as an antioxidant, there is even a claim that gout may be one of the most important antioxidants in plasma. Uric acid works in the form of urate, which is a form of uric acid that dissolves in the blood, urate will react with various oxidants, such as hydrogen peroxide, hydroxyl radicals, and nitric oxide (NO) derivatives, and can neutralize the toxic effects of these oxidants (Roumeliotis et al., 2019). This further reinforces that high SAT in CKD patients is affected by gout.

High TAS in HD patients can also be influenced by extrinsic factors such as the type of dialyzer membrane used and antioxidant interventions before HD, during HD, and after HD. In this study, dialyzer membrane used was a bioincompatible dialyzer membrane, thus the total antioxidant status would decrease due to its exposure to blood which would result in an increase in oxidative stress. In the study, there was no data search on what interventions were given to the two groups of subjects, it was because the non-HD group subjects were not given specific directions on their diets, whereas in HD subjects there were no data on antioxidant interventions whether they were food or supplement. Serum taking factors also influence high TAS in patients with CKD HD. In this study, serum subjects with CKD undergoing HD were taken before routine $\mathrm{HD}$ was performed, whereas routine $\mathrm{HD}$ was performed 2 times/week. According to Mayer et al. (2003), serum intake time affected TAS in patients with HD $\mathrm{CKD}$, which in his study it was found that TAS levels during the HD process would decrease dramatically and only about $28 \%$ of the total antioxidant would occur during the initial HD procedure. This supports that serum intake time affects TAS levels.

Although there are many factors that can exacerbate oxidative stress, when viewed from data on the types of antioxidants and oxidative stress biomarkers found in both groups of subjects, it is found that 
antioxidants in the form of albumin are still higher when compared to oxidative stress biomarkers in the form of MDA, this might also apply to other antioxidants found in the bodies of both groups of subjects, thus the amount of all antioxidants would be higher than the amount of all oxidative stress, thus TASs obtained would be of high value, especially in CKD patients undergoing HD procedures, this was due by the treatment of these HD procedures and the interventions given.

\section{CONCLUSION}

Total antioxidant status in CKD patients undergoing HD is higher compared to non-HD CKD patients. This is caused by the treatment received by CKD patients who underwent HD before, during, and after the HD procedure was performed, as well as random serum intake time. In this study, serums were taken before routine HD, which allowed an accumulation of antioxidants in the body before HD. Further research by examining antioxidants and oxidative stress as a whole that can affect TAS levels in CKD patients; serum extraction is done before, during and after the HD procedure; as well as conducting a comprehensive data collection starting from the antioxidant intervention obtained by the patient until all components regarding hemodialysis need to be done to be able to further confirm which TAS levels are higher.

\section{REFERENCES}

Almansa, I., Barcia, J.M., López-Pedrajas, R., Muriach, M., Miranda., Romero, F.J., 2013. Naltrexone reverses ethanol-induced rat hippocampal and serum oxidative damage. Oxidative Medicine and Cellular Longevity. 2013

Berns, J.S, 2018. Patient education: Hemodialysis (Beyond the Basics). Downloaded 4 May 2018 from https://www.uptodate.com/contents/hemod ialysis-beyond-the-basics/print

Buico, A., Cassino, C., Ravera, M., Betta, P., Osella, D., 2009. Oxidative stress and total antioxidant capacity in human plasma. Redox Report, 14(3): 125-31.

Cohen, G., Hörl, W.H., 2012. Immune dysfunction in uremia. An update. Toxins, 4(11): 962-90.

Coombes, J.S., Fassett, R.G., 2012. Antioxidant therapy in hemodialysis patients: A systematic review. Kidney International, 81(3): 233-46.

Delaney, M.P., Price C.P., Newman D.J., 2006. Kidney disease. In: E. Lamb, ed. Tietz Texstbook of Clinical Biochemistry. 4th ed. Saunder. 1670-1745..

Filiopoulos, V., Hadjiyannakos, D., Takouli, L., Metaxaki, P., Sideris, V., Vlassopoulos, D., 2009. Inflammation and oxidative stress in end-stage renal disease patients treated with hemodialysis or peritoneal dialysis. Int J Artif Organs, 32(12): 872-82.

Flores-Bellver, M., Bonet-Ponce, L., Barcia, J. et al. 2014. Autophagy and mitochondrial alterations in human retinal pigment epithelial cells induced by ethanol: implications of 4-hydroxy-nonenal. Cell Death Dis, 5: e1328

Goldberg H.J., Scholey J., Fantus I. G., 2000. Glucosamine activates the plasminogen activator inhibitor 1 gene promoter through Sp1 DNA binding sites in glomerular mesangial cells. Diabetes, 49(5): 86371.

Kidney Health Australia, 2015. Albuminuria. Downloaded 20 October 2018 from https://kidney.org.au/cms_uploads/docs/al buminuria-fact-sheet.pdf

Kuo, K.L., Tarng, D.C., 2010. Oxidative stress in chronic kidney disease. Adaptive Medicine, 2(2), 8794.

Labriola, L., Jadoul, M., 2017. Dialysis-related amyloidosis: Is it gone or should it be? Seminars in Dialysis. 30(3):193-6.

Ladi-Akinyemi, T.W., Ajayi, I., 2017. Risk factors for chronic kidney disease among patients at Olabisi Onabanjo University Teaching Hospital in Sagamu, Nigeria: a retrospective cohort study. Malawi Med J, 29(2), 166-70.

Liakopoulos, V., Roumeliotis, S., Gorny, X., Dounousi, E., Mertens, P.R., 2017. Oxidative stress in hemodialysis patients: a review of the literature. Oxidative Medicine and Cellular Longevity, 2017.

Liguori, I., Russo, G., Curcio, F., Bulli, G., Aran, L., Della-Morte, D., Gargiulo, G., Testa, G., Cacciatore, F., Bonaduce, D., Abete, P., 2018. Oxidative stress, aging, and diseases. Clinical Interventions in Aging, 13: 757-72. 
Mayer, B., Zitta, S., Greilberger, J., Holzer, H., Reibnegger, G., Hermetter, A., Oettl, K., 2003. Effect of hemodialysis on the antioxidative properties of serum. Biochimica et Biophysica Acta (BBA) Molecular Basis of Disease, 1638(3): 267-72.

Medscape, 2018. Do I need a microalbumin urine test? Downloaded 22 October 2018 from https://www.webmd.com/diabetes/urine- test-microalbumin\#2

Montazerifar, F., Karajibani, M., Sanadgol, H., Hashemi, M., 2012. Effect of peritoneal dialysis on antioxidant defense system and oxidative stress. Hong Kong Journal of Nephrology, 14(2): 33-7.

NIDDK, 2018. Albuminuria: Albumin in the urine. Downloaded 22 October 2018 from https://www.niddk.nih.gov/health-information/kidney-disease/chronic-kidney-disease-ckd/testsdiagnosis/albuminuria-albumin-urine

Nsonwu-Anyanwu, A., Offor, S., John, I., 2018. Cigarette smoke and oxidative stress indices in male active smokers. Reactive Oxygen Species, 5(15): 199-208.

Putri, A.Y., Thaha, M., 2014. Role of oxidative stress on chronic kidney disease progression,.Acta Medica Indonesiana, 46(3): 244-52.

Roumeliotis, S., Roumeliotis, A., Dounousi, E., Eleftheriadis, T., Liakopoulos, V. 2019. Dietary antioxidant supplements and uric acid in Chronic Kidney Disease: A Review. Nutrients, 11(8): 1911.

Sheen, Y.J., Sheu, W.H.H., 2011. Metabolic syndrome and renal injury. Cardiology Research and Practice, 2011: 1-13.

Sundaram, M.S., Nagarajan, S., Jagdeeshwaran, A., Devi, Manjula A.J., 2014. Chronic kidney disease - effect of oxidative stress. Chinese Journal of Biology, 2014: 1-6.

Tajbakhsh, R., Qorbani, M., Mehrpour, G., Rahimzadeh, M., Azimzadeh., M.M., Mirmiranpour, H., 2017. Effect of hemodialysis on oxidants and antioxidant factors in chronic renal failure. Saudi Journal of Kidney Diseases and Transplantation, 28(3): 507-16.

Tangvarasittichai, S., 2015. Oxidative stress, insulin resistance, dyslipidemia and type 2 diabetes mellitus. World Journal of Diabetes, 6(3): 456.

Thaha, M., Empitu, M.A., Kadariswantiningsih, I.N., Santoso, D., Tomino, Y., 2017. [Untitled work]. Unpublished raw data.

van der Vaart, H., Postma, D.S., Timens, W., Hylkema, M.N., Willemse, B.W.M., Boezen, H.M., Vonk, J.M., de Reus, D.M., Kauffman, H.F., ten H., Nick, H.T., 2005. Acute effects of cigarette smoking on inflammation in healthy intermittent smokers. Respiratory Research, 6(1):22. 